\title{
A CORRELATION-DRIVEN MAPPING FOR DEEP LEARNING APPLICATION IN DETECTING ARTIFACTS WITHIN THE EEG
}

\author{
Nooshin Bahador, Kristo Erikson, Jouko Laurila, Juha Koskenkari, Tero Ala-Kokko, Jukka \\ Kortelainen
}

\begin{abstract}
Objective: When developing approaches for automatic preprocessing of electroencephalogram (EEG) signals in non-isolated demanding environment such as intensive care unit (ICU) or even outdoor environment, one of the major concerns is varying nature of characteristics of different artifacts in time, frequency and spatial domains, which in turn causes a simple approach to be not enough for reliable artifact removal. Considering this, current study aims to use correlation-driven mapping to improve artifact detection performance.

Approach: A framework is proposed here for mapping signals from multichannel space (regardless of the number of EEG channels) into two-dimensional RGB space, in which the correlation of all EEG channels is simultaneously taken into account, and a deep convolutional neural network $(\mathrm{CNN})$ model can then learn specific patterns in generated 2D representation related to specific artifact.

Main results: The method with a classification accuracy of $92.30 \%$ (AUC=0.96) in a leave-three-subjects-out cross-validation procedure was evaluated using data including 2310 EEG sequences contaminated by artifacts and 2285 artifact-free EEG sequences collected with BrainStatus self-adhesive electrode and wireless amplifier from 15 intensive care patients. For further assessment, several scenarios were also tested including performance variation of proposed method under different segment lengths, different numbers of isoline and different numbers of channel. The results showed outperformance of CNN fed by correlation coefficients data over both spectrogram-based $\mathrm{CNN}$ and EEGNet on the same dataset.

Significance: This study showed the feasibility of utilizing correlation image of EEG channels coupled with deep learning as a promising tool for dimensionality reduction, channels fusion and capturing various artifacts patterns in temporal-spatial domains. A simplified version of proposed approach was also shown to be feasible in real-time application with latency of $0.0181 \mathrm{~s}$ for making real-time decision.
\end{abstract}

Keywords- Deep Learning, Multichannel Data Fusion, Dimensionality Reduction, CNN, Correlation Map, EEG, Artifact, Real-Time Application.

\section{INTRODUCTION}

Electroencephalogram (EEG) is a measure of the electrical activity of the brain mainly arising from the cortical synaptic action. However, these signals are usually contaminated with different unwanted artifacts during the recording, seriously affecting the interpretation of the signal and require to be eliminated before further analysis. The artifacts are

Orion Pharma is gratefully acknowledged for the unrestricted financial support of this study.

N. Bahador is with the Physiological Signal Analysis Team, Center for Machine Vision and Signal Analysis, MRC Oulu, University of Oulu, Oulu, Finland (E-mail: nooshin.bahador@oulu.fi).

K. Erikson, J. Laurila, J. Koskenkari and T. Ala-Kokko are with the Research Group of Surgery, Anaesthesiology and Intensive Care, Medical Faculty, University of Oulu, Oulu, Finland and with the Division of Intensive substantially present in the recordings of critically ill patients due to the long duration of the measurement and challenges related to the environment such as the presence of electromechanical devices as well as motion artifacts arising from both staff and patient [1-4].

Detecting artifacts automatically from EEG recordings has proven to be a challenging task. Simple amplitude- or frequency-based methods have not succeeded in this due to the varying characteristics of both, the artifacts themselves as well as the background EEG activity. Consequently, several more advanced methods have been proposed in literature. These methods have, however, limitations such as requirements related to specific features of the artifacts (such as spike shapes, number of spikes, peak-to-peak amplitude, relative amplitude, peak location in channels, signal power in the entire window, maximum power difference between adjacent segments etc.) or using of a reference signals like separate channel for ECG or EOG [5-11].

Recent advances in machine learning methods have increasingly captured the attention for distinguishing artifactfree EEG sequences from contaminated ones [12-17]. So far, there has been a limited number of studies focusing on a fully automatic removal method based on deep learning and the proposed approaches only focus on specific types of artifacts which leads to limited generalization of the method to the artifacts resulting from various sources [18, 19]. Moreover, most of the studies have been using recordings made with a full EEG cap which is challenging in the intensive care environment where securely maintaining electrode contacts and easy longterm high-quality signal acquisition is beneficial.

Current work presents a quantitative technique for artifact detection by integrating information from different channels of EEG based on correlation coefficient and needing no auxiliary reference signal. In this method, correlation coefficient matrix of all channels is formed as contour map and fed to a deep network to classify if an EEG sequence includes artifact or not. The proposed method enables detection of various types of artifact with different patterns in both amplitude and frequency. Additionally, this study explores and validates the performance

Care Medicine, MRC Oulu, University of Oulu and Oulu University Hospital, Oulu, Finland.

J. Kortelainen is with the Physiological Signal Analysis Team, Center for Machine Vision and Signal Analysis, MRC Oulu, University of Oulu, Oulu, Finland and with the Cerenion Oy, Oulu, Finland. 
of the proposed deep learning-based method for artifact detection from recordings made with an easy-to-use forehead EEG electrode and wireless device suitable for demanding clinical environment such as intensive care unit (ICU).

\section{DATA COLLECTION}

The EEG dataset used in this study was collected from 15 patients treated in the ICU of Oulu University Hospital. The patients had no history of serious neurological disease and were 18-85 years in age. During the recording, the patients were not mechanically ventilated and were recently diagnosed with hyperactive delirium. Delirium was treated with administration of dexmedetomidine following the ICUs standard protocol to keep the patients moderately sedated. The study was approved by local ethics committee. Written informed consent for participation was obtained either from the patient or his/her relative.

The EEG data was recorded with the BrainStatus selfadhesive electrode (Figure 1) and BrainStatus wireless amplifier [23] with a sampling frequency of $250 \mathrm{~Hz}$. The EEG recordings contained 10 channels (Sp2, T10, Af8, F8, Fp2, Fp1, T9, F7, Sp1, AF7). From the recordings, 4595 non-overlapping 30-s segments were extracted offline. Segments were picked so that 2310 of them contained artifacts and others were artifactfree. The segments were manually inspected and labeled based on visual inspection.

The sequences containing high amplitude and sharply contoured transients distinguishable from background activity were classified to contain EMG artifact. Those sequences having high amplitude and low frequency activity with morphology of steep fall followed by the slower rise were classified to contain EOG artifact. High amplitude sharp waves were also considered as transient artifact. Sequences with nonlinear curved pattern which leads to continuous decrease or increase in amplitude over time was categorized to have trend artifact. Relatively high amplitude single-frequency harmonic noise was also considered as powerline interference artifact. Figure 2 shows sample sequences in the dataset with the abovementioned different artifacts in all channels.

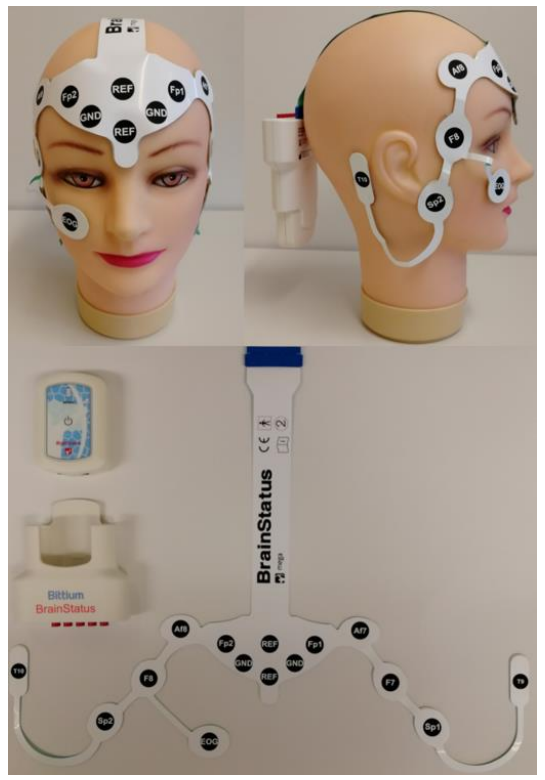

Fig. 1. EEG electrode set and device used for data collection

\section{Method}

\subsection{Proposed Method}

As already discussed, not only the EEG amplitude may change a lot especially in the ICU environment under varying conditions, but also the artifact characteristics including the amplitude vary a lot as well, and this issue causes a simple approach (e.g. based on thresholding) to be not enough for reliable artifact detection. Therefore, a more sophisticated technique is required for precisely detecting the artifacts. To address this issue by taking advantage of deep learning, the current study introduced a computationally efficient artifact detection method using interpreting the multidimensional information and extracting a meaningful knowledge of the raw data coming from multiple channels. This proposed information fusion automatically transforms data from different channels in time into a single $2 \mathrm{D}$ representation that provides fast effective

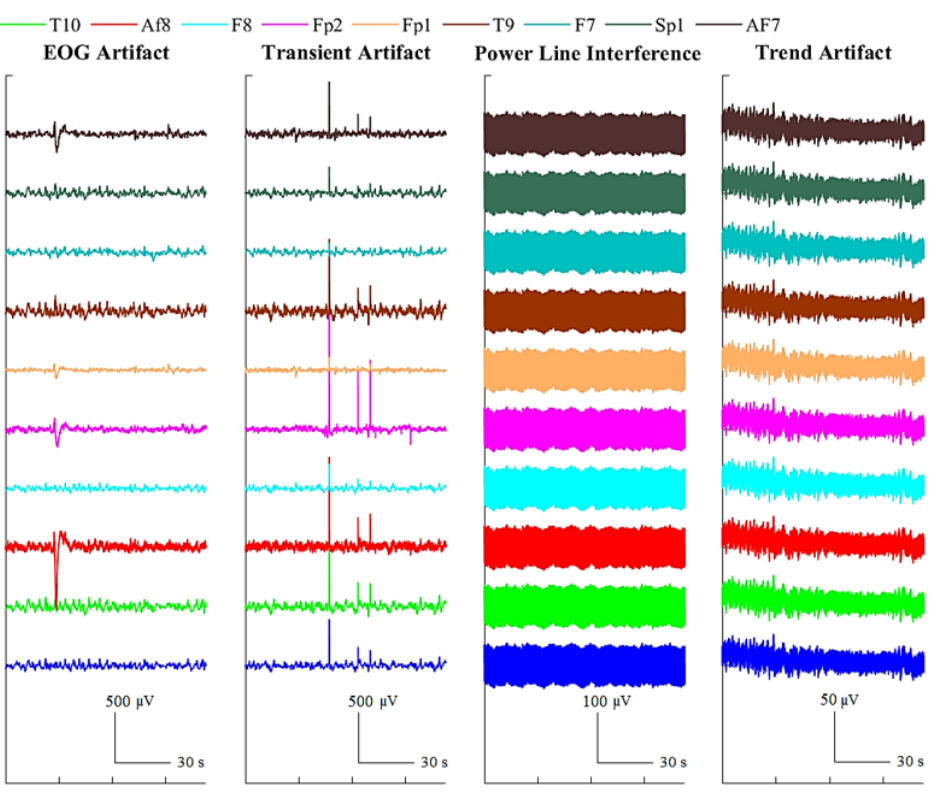

Fig. 2. Different types of artifact within studied dataset support for discriminating artifact-contaminated segments from others. The idea of this method is on the hypothesis that data driven by different channels has correlation with each other and correlation matrix of all these measurements has a unique distribution associated with each type of artifact which can be visualized as a contour plot. Generating these contour images is the preprocessing phase of proposed method shown in figure 3 . Given with 2D correlation contour as input data sets, a deep convolutional neural network $(\mathrm{CNN})$ model can learn specific patterns in 2D correlation representation related to specific artifact. As shown in figure 3, In second phase, the training set of input-label pairs is utilized to train the CNN model based on initial parameters and then update them via minimizing the loss function. In validation phase, a validation set is used to evaluate the predictor. Below is the summary of the steps followed in the method: 
Step 1- Computing the correlation coefficient of two channels of $\mathrm{i}$ and $\mathrm{j}$ according to Equation (1).

$$
\begin{aligned}
& \rho\left(S_{i}, S_{j}\right)=\frac{1}{n-1} \sum_{k=1}^{n}\left(\frac{S_{k}-\mu_{i}}{\sigma_{i}}\right)\left(\frac{S_{k}-\mu_{j}}{\sigma_{j}}\right) \\
& \rho\left(S_{i}, S_{j}\right)=\frac{\operatorname{cov}\left(S_{i}, S_{j}\right)}{\sigma_{i} \sigma_{j}}
\end{aligned}
$$

where $\mu_{\mathrm{i}}$ and $\sigma_{\mathrm{i}}$ are the mean and standard deviation of $\mathrm{S}_{\mathrm{i}}$, respectively, and $\mu_{\mathrm{j}}$ and $\sigma_{\mathrm{j}}$ are the mean and standard deviation of $S_{j}$.

Step 2- Obtaining the correlation coefficient matrix of all channels according to Equation (2).

$$
\mathrm{R}=\left(\begin{array}{ccc}
\rho\left(\mathrm{S}_{1}, \mathrm{~S}_{1}\right) & \cdots & \rho\left(\mathrm{S}_{1}, \mathrm{~S}_{\mathrm{n}}\right) \\
\vdots & \ddots & \vdots \\
\rho\left(\mathrm{S}_{\mathrm{n}}, \mathrm{S}_{1}\right) & \cdots & \rho\left(\mathrm{S}_{n}, \mathrm{~S}_{\mathrm{n}}\right)
\end{array}\right)
$$

Step 3-Creating a filled contour plot containing the isolines of obtained matrix of R.

Step 4- Feeding contour plot to the deep network to classify the sequences with artifact.

Figure 4 and 5 show the preprocessing steps performed on the raw EEG data in order to prepare input data for $2 \mathrm{D}$ convolutional neural network (CCN). As shown in figure 4, correlation coefficients between channels was first derived and presented in the form of contour map. For comparison phase, the spectrogram of the EEG sequence was also generated for each channel (figure 5), and mean value of obtained images of whole channels was then calculated. The obtained images were separately fed to a deep network as input image. While this correlation contour image summarizes what occurs in temporalspatial domain, the mean spectrogram represents what happens in time-frequency domain.

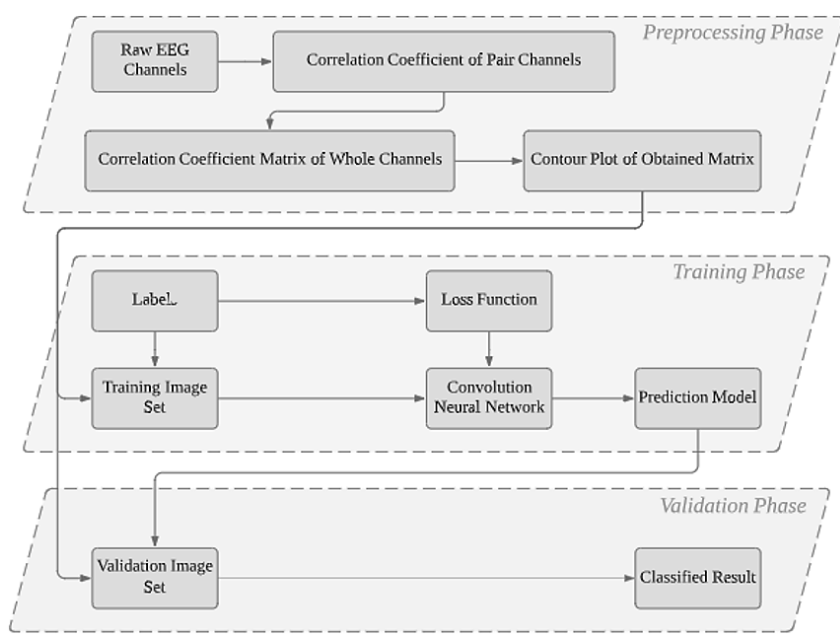

Fig. 3. Block diagram of the proposed method
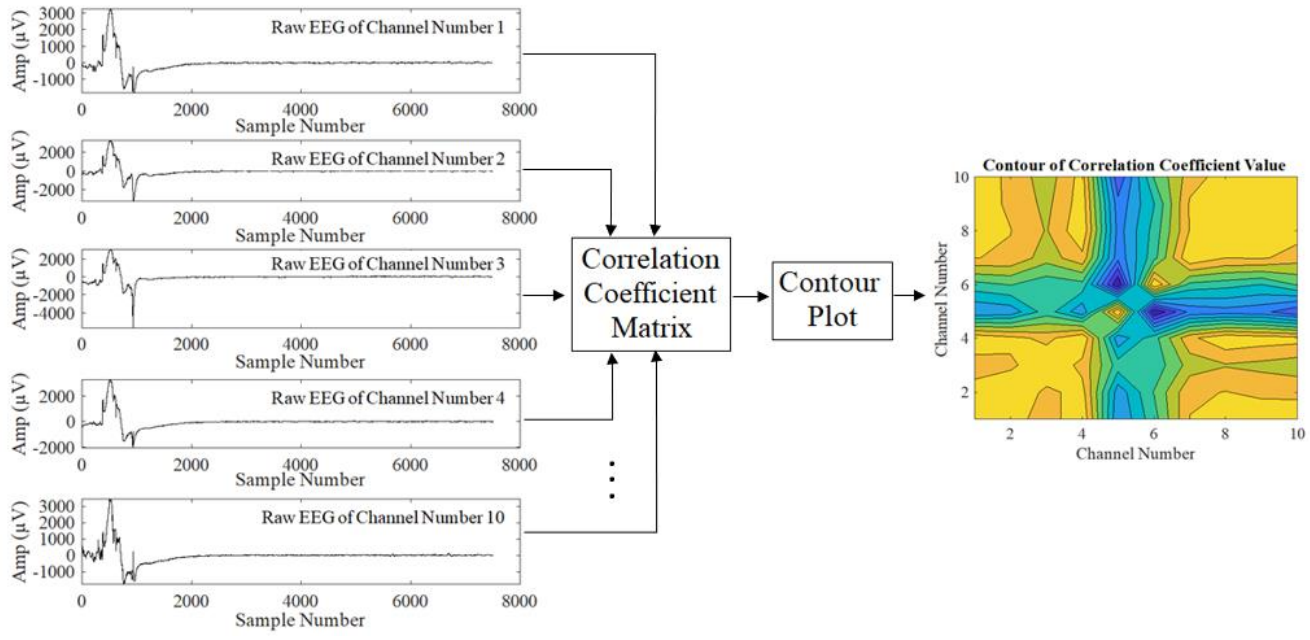

Fig. 4. Generating correlation coefficients contour
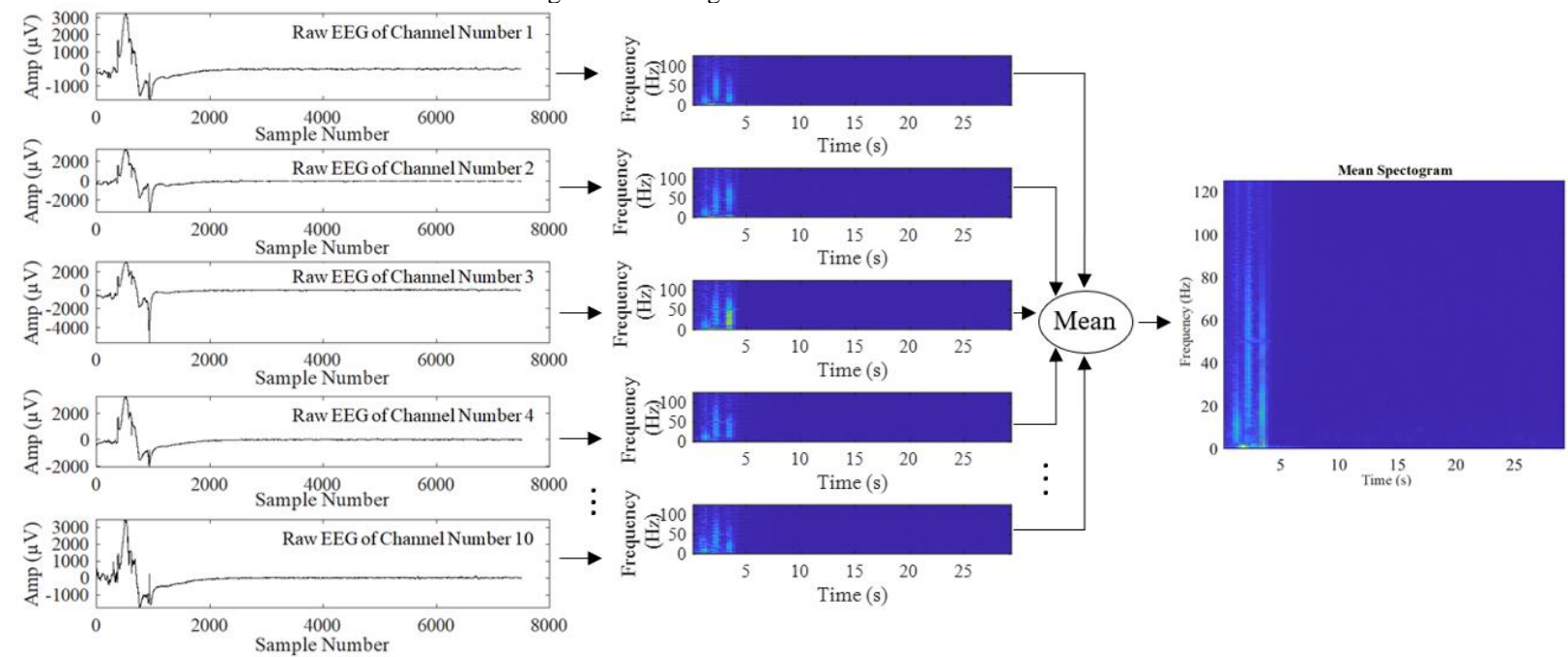

Fig. 5. Generating mean spectogram 
The proposed deep learning architecture for image-to-label classification is presented in figure 6 and consisted of a CNN with three two-dimensional convolution layers, followed by batch normalization, Relu, max pooling and fully connected layers. The 2D convolutional layer applied sliding convolutional filters to the input contour image. The output of this network is a categorical response and therefore a Softmax and classification layers was also added as last layers of each CNN. All layers in each CNN were connected sequentially. Final layer of the whole network is decision layer of this CNN classifiers.

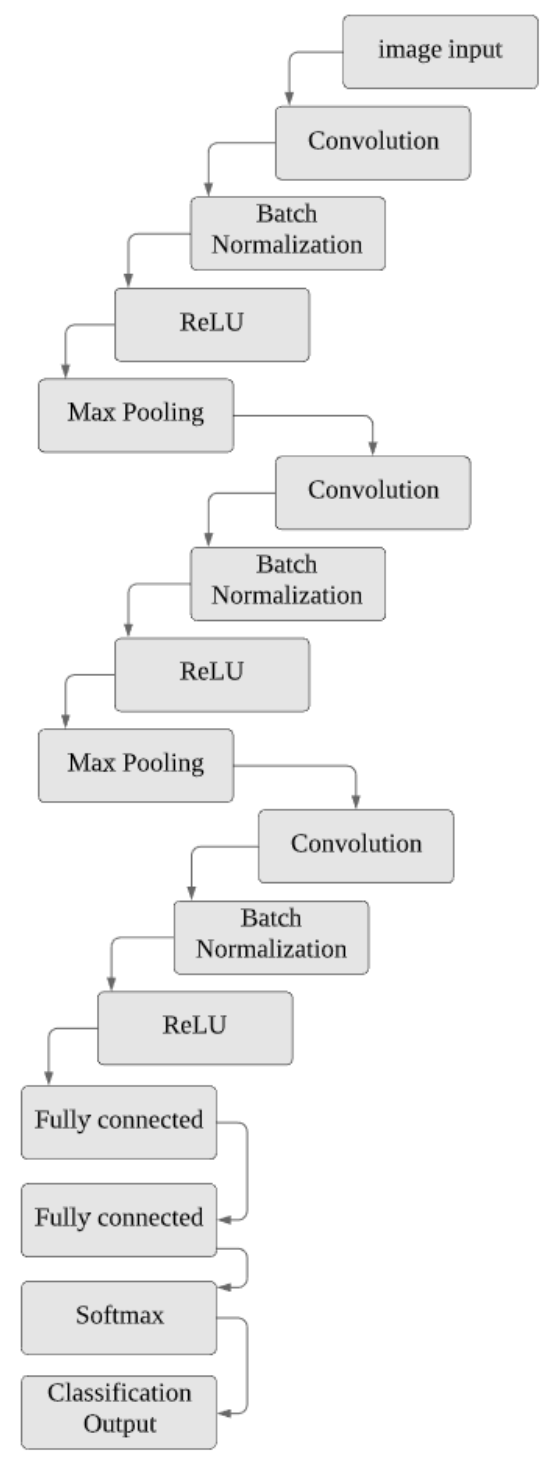

Fig. 6. $\mathrm{CNN}$ architecture

The training parameters of deep learning model are given in Table 1. The mini-batch size and the maximum number of epochs were respectively set to 32 and 5 . These small minibatches with short sequences were used to make it more suitable for training on the CPU. Leave-three-subjects-out crossvalidation was also used to check the performance of model in which three subjects were chosen for testing while the other 12 subjects were applied for training. These steps were repeated until all the subjects have been used as test group.

\begin{tabular}{lcll}
\multicolumn{1}{c}{ TABLE I. } & \multicolumn{2}{c}{ THE MODEL TRAINING PARAMETERS } \\
\hline \hline Parameter & Value & Parameter & Value \\
\hline \hline Initial Learn Rate & 0.001 & Mini Batch Size & 32 \\
Learn Rate Drop Factor & 0.05 & Max Epochs & 5 \\
Learn Rate Drop Period & 2 & Learn Rate Schedule & Piecewise \\
\hline \hline
\end{tabular}

\subsection{Summary of the Analysis Conducted}

The above-described analysis provides us with the following results:

1- Correlation coefficient contour comparison for sequences with and without artifacts: This analysis was designed to visualize difference in distribution of correlation maps and their color patterns across the subjects for artifact-free and artifact-contaminated sequences.

2- The effect of parameter selection on correlation coefficients contour: This analysis determines whether the patterns in correlation maps are independent of involved parameters.

3- Performance comparison between correlation-based CNN and spectogram-based CNN: This analysis tests whether CNN fed by correlation map outperforms the $\mathrm{CNN}$ fed by spectrogram.

4- Performance comparison between correlation-based CNN and EEGNet: This analysis was designed to test whether CNN fed by correlation map outperforms EEGNet fed by raw EEG signals.

5- The effect of parameter selection on the results of correlation-based CNN: This analysis was designed to see if the changes in parameter values affect classification performance.

6- Real-time application: This analysis was conducted to determine whether using a simplified $\mathrm{CNN}$ fed by lowresolution version of correlation map could make this approach applicable for real-time computing.

\section{RESULTS \\ 4.1. CORRELATION COEFFICIENTS CONTOUR COMPARISON FOR SEQUENCES WITH AND WITHOUT ARTIFACTS.}

From the color histograms of correlation maps in all subjects (figure 7), normal sequences have roughly different distribution of colors from sequences contaminated by artifacts. Figure 8 and 9 show examples of correlation coefficients contour generated from corresponding EEG sequences including 10 channels. The horizontal and vertical axis represents channel number. The value of correlation coefficients is represented by the color, with dark colors corresponding to low values and bright colors corresponding to high values. As seen in figures, there is a visible difference in the color patterns of correlation coefficients contour for EEG sequences with and without artifacts. 


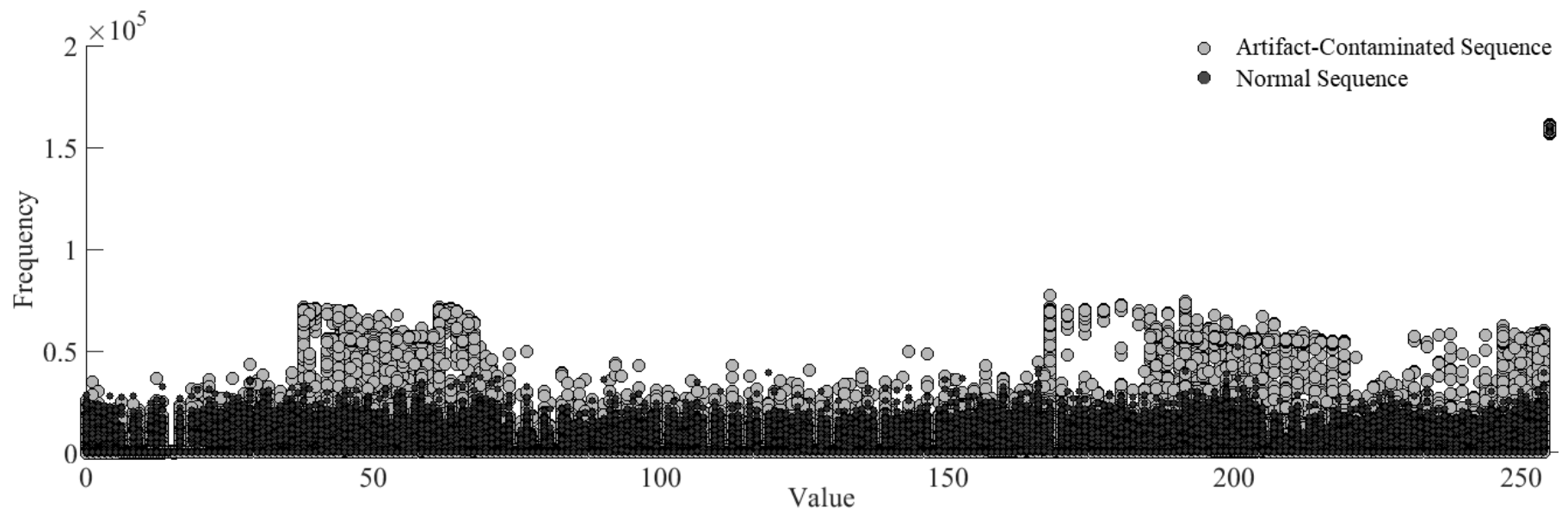

Fig. 7. Distribution of colors in correlation maps of all subjects

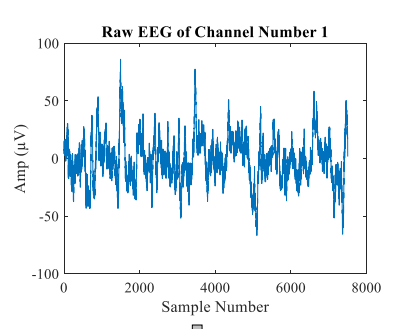

囚

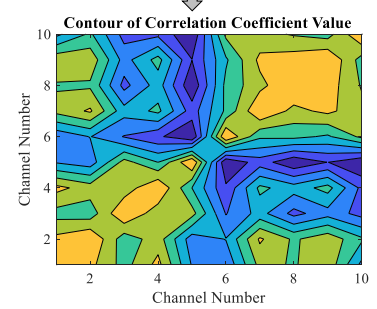

Fig.8.1. Sequence 1
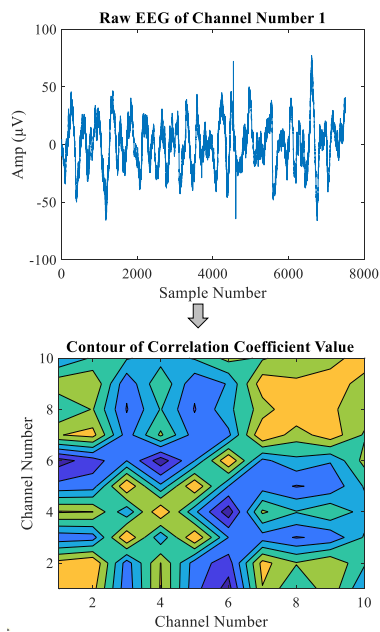

Fig.8.2. Sequence 2
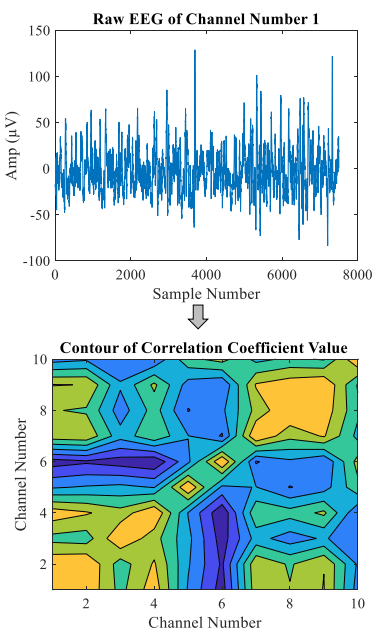

Fig.8.3. Sequence 3

Fig.8. Examples of correlation coefficients contour for artifact-free EEG sequences

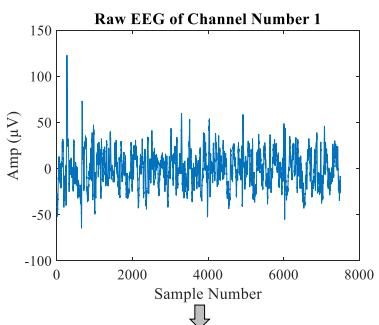

$\Omega$

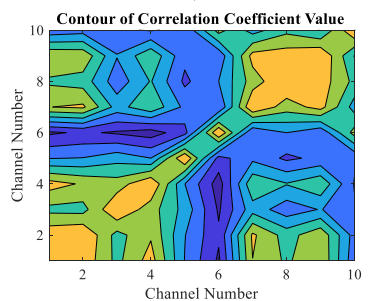

Fig.8.4. Sequence 4

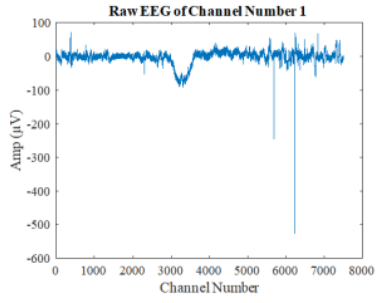

$\sqrt{3}$

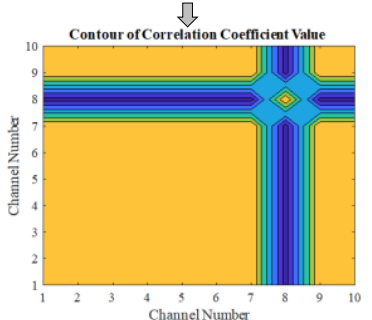

Fig.9.1. Sequence 1

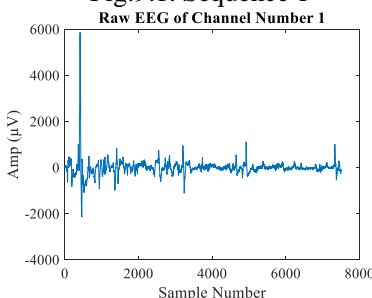

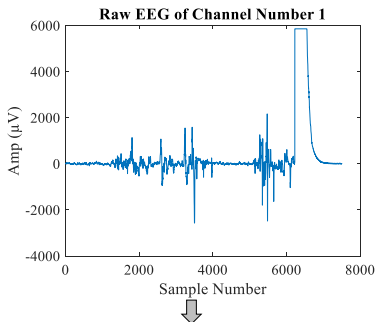

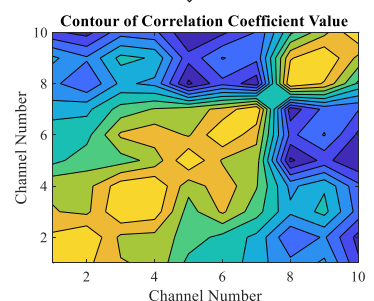

Fig.9.2. Sequence 2

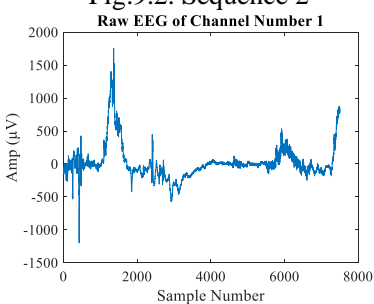

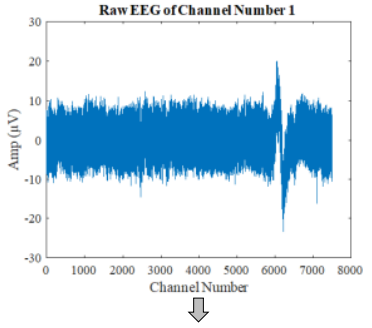

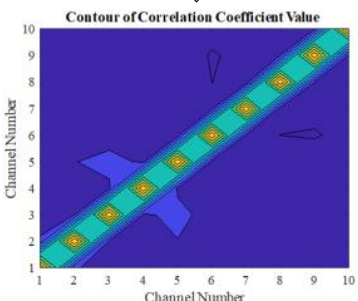

Fig.9.3. Sequence 3

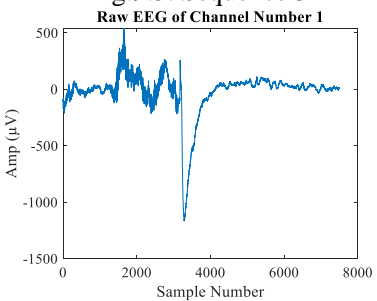

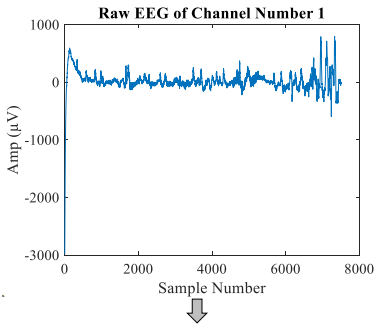

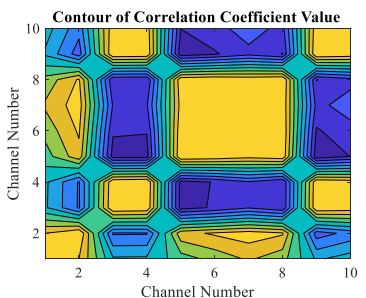

Fig.9.4. Sequence 4

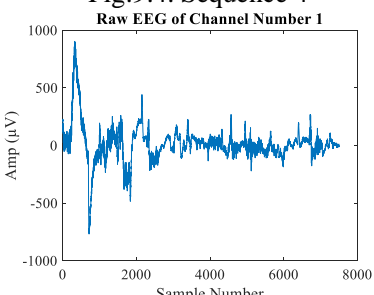


\&

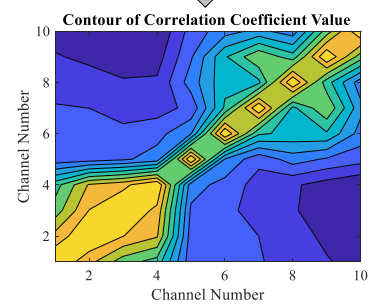

Fig.9.5. Sequence 5
$\S$

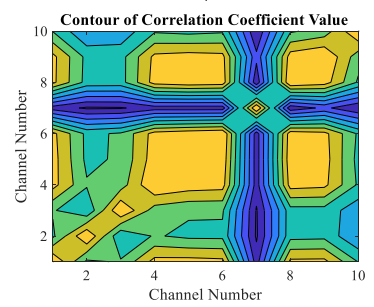

Fig.9.6. Sequence 6 $\sqrt{3}$

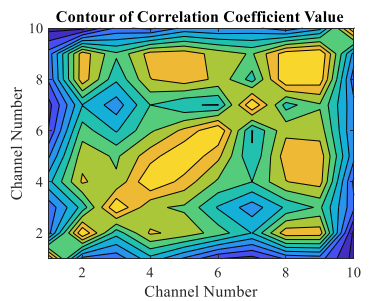

Fig.9.7. Sequence 7

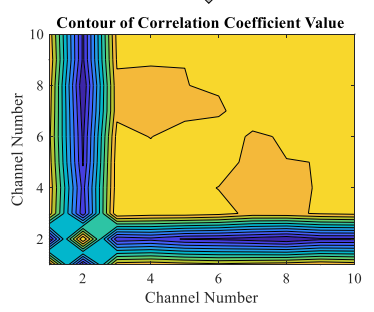

Fig.9.8. Sequence 8

Fig.9. Examples of correlation coefficients contour for EEG sequences including artifacts

4.2. THE EFFECT OF PARAMETER SELECTION ON CORRELATION COEFFICIENTS CONTOUR

There are several important parameters considered in evaluating performance including segment length, number of isolines and number of channels where their corresponding correlation coefficients contour are respectively shown in figure
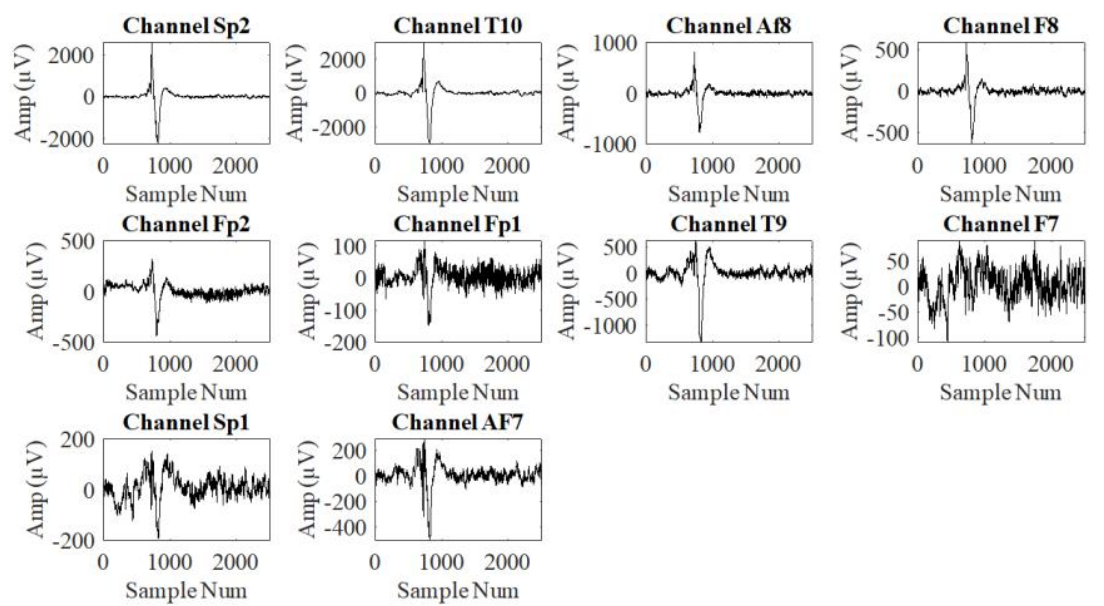

10 to 12 . From these figures, it could be concluded that most patterns in correlation coefficients contour are somehow independent of both segment length and the number of isoline. However, applying few numbers of channel leads to slightly different patterns in correlation coefficients contour.
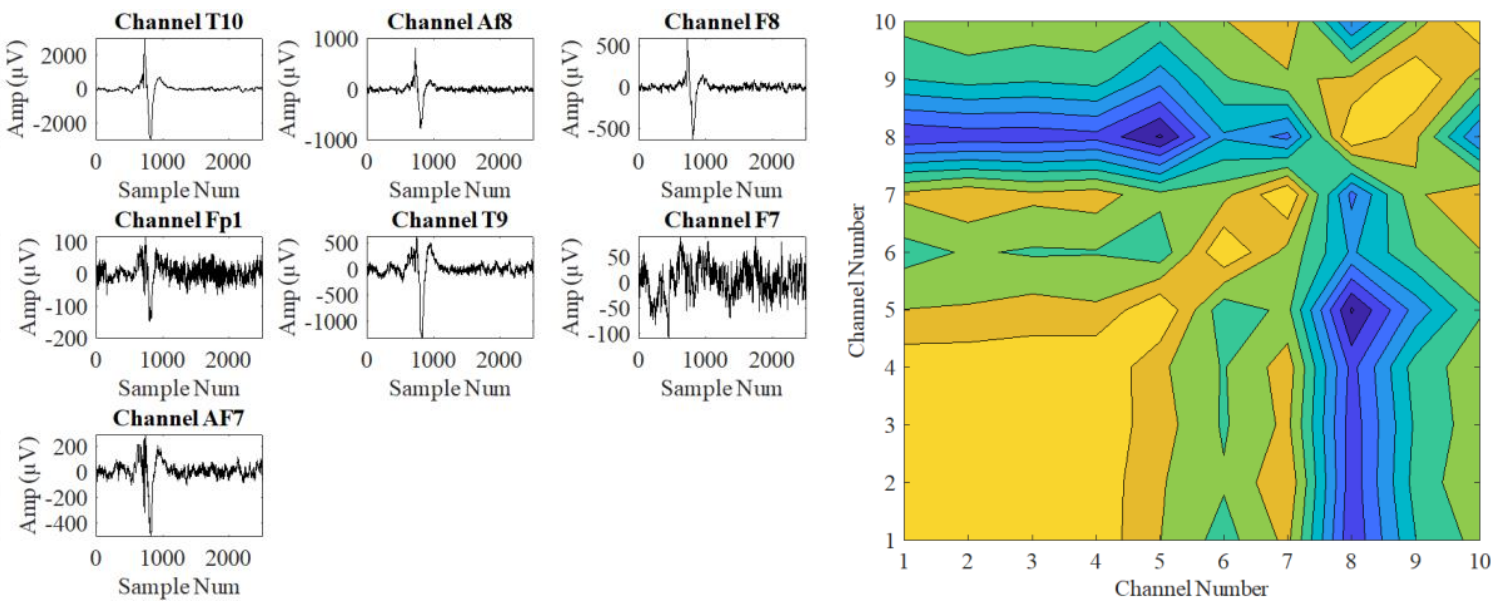

Fig.10.1. Segments length of $10 \mathrm{~s}$
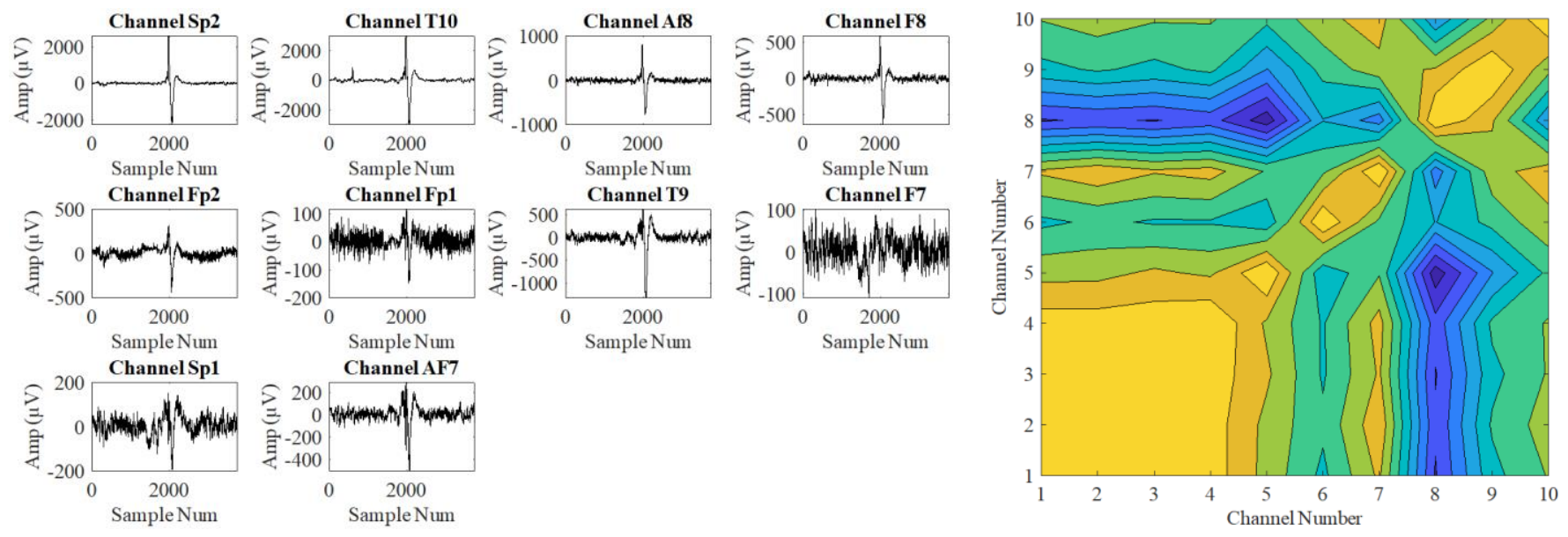

Fig.10.2. Segments length of $15 \mathrm{~s}$ 

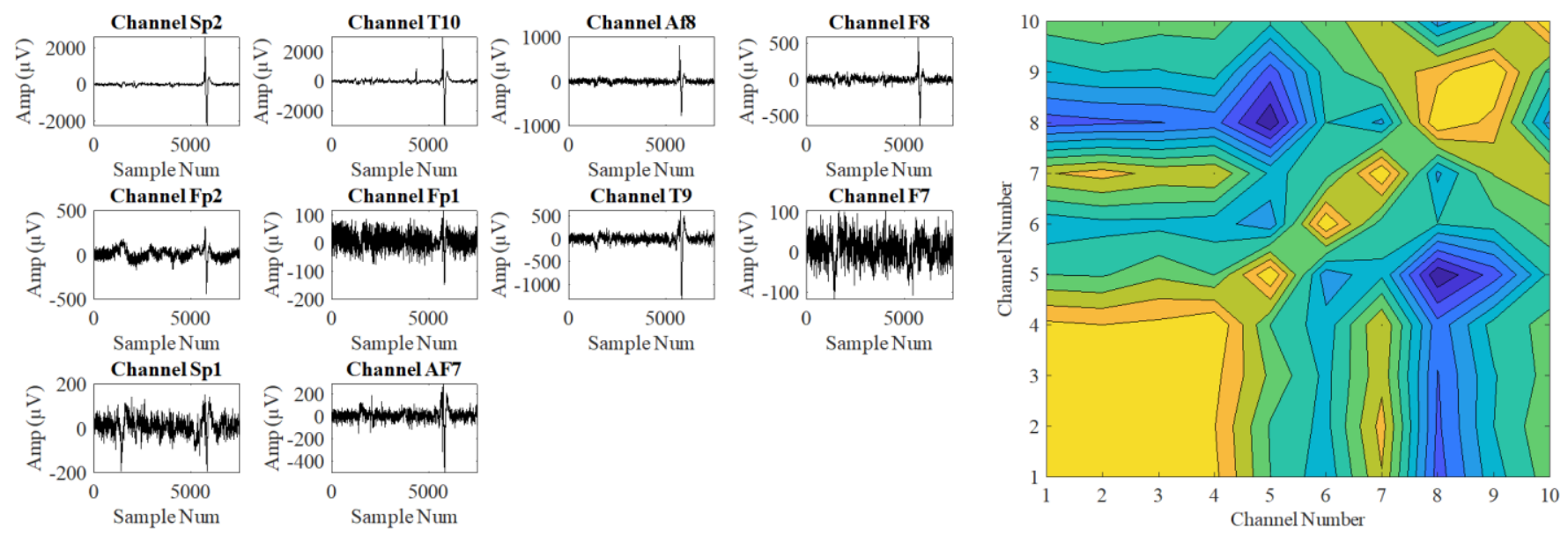

Fig.10.3. Segments length of $30 \mathrm{~s}$

Fig.10. The effect of different segments lengths in patterns of correlation coefficient contour plot

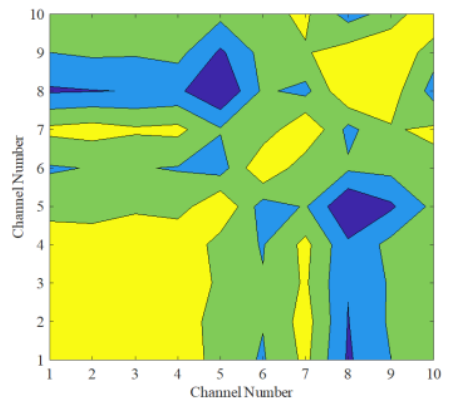

Fig.11.1. Isoline number of 3

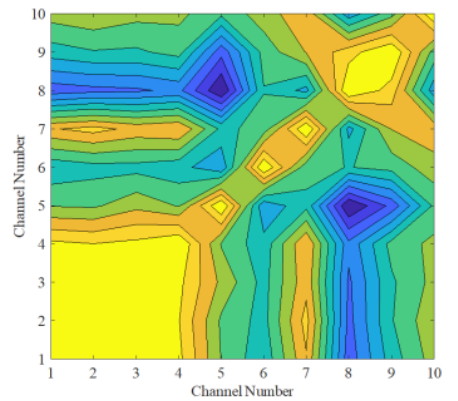

Fig.11.4. Isoline number of 10

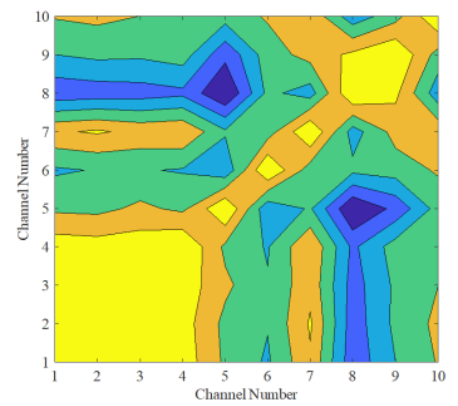

Fig.11.2. Isoline number of 5

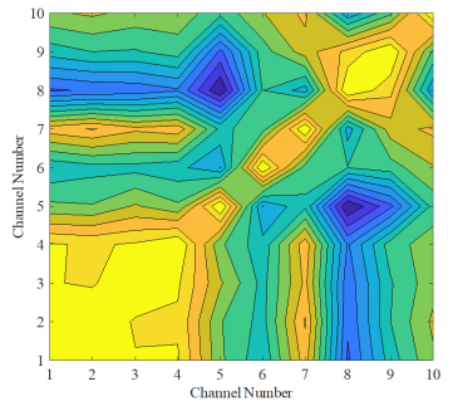

Fig.11.5. Isoline number of 12

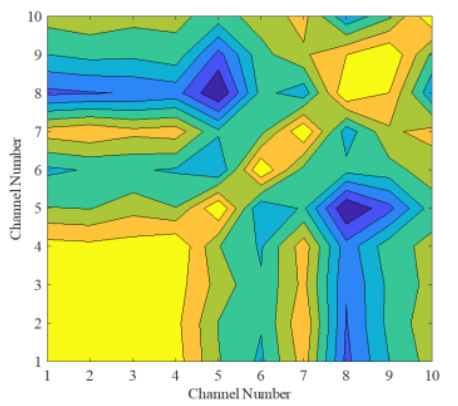

Fig.11.3. Isoline number of 7

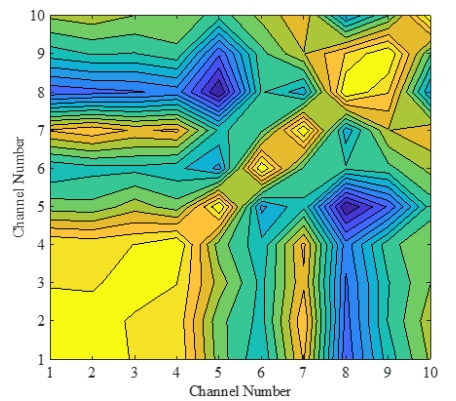

Fig.11.6. Isoline number of 14

Fig.11. The effect of different isoline numbers in patterns of correlation coefficient contour plot
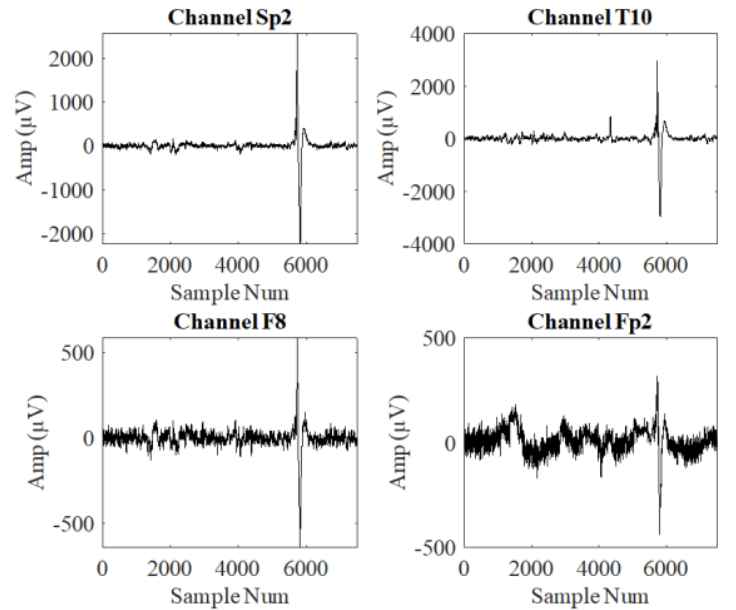
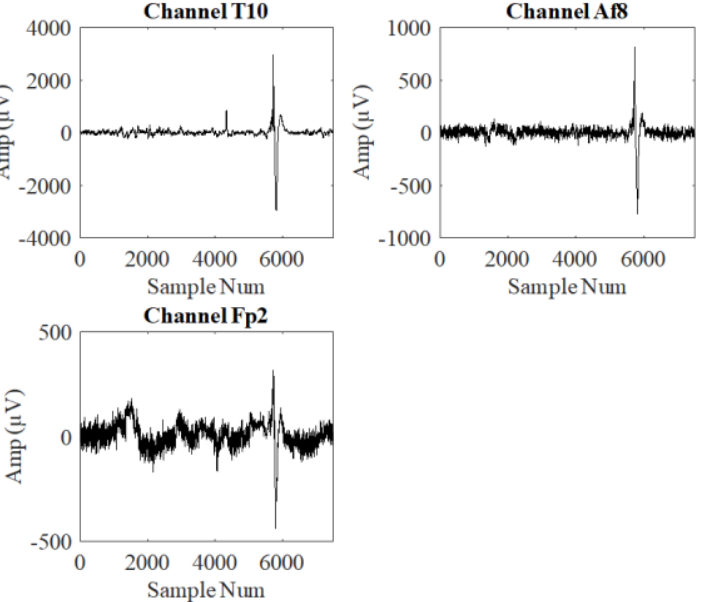

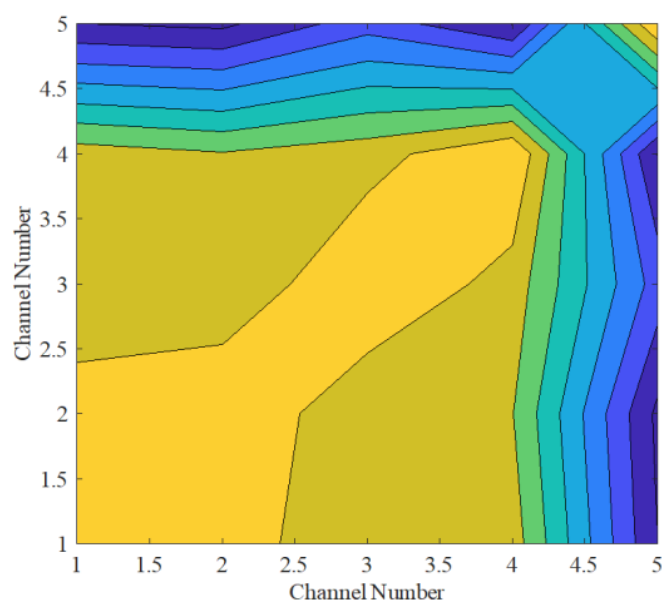

Fig.12.1. Channel number of 5 

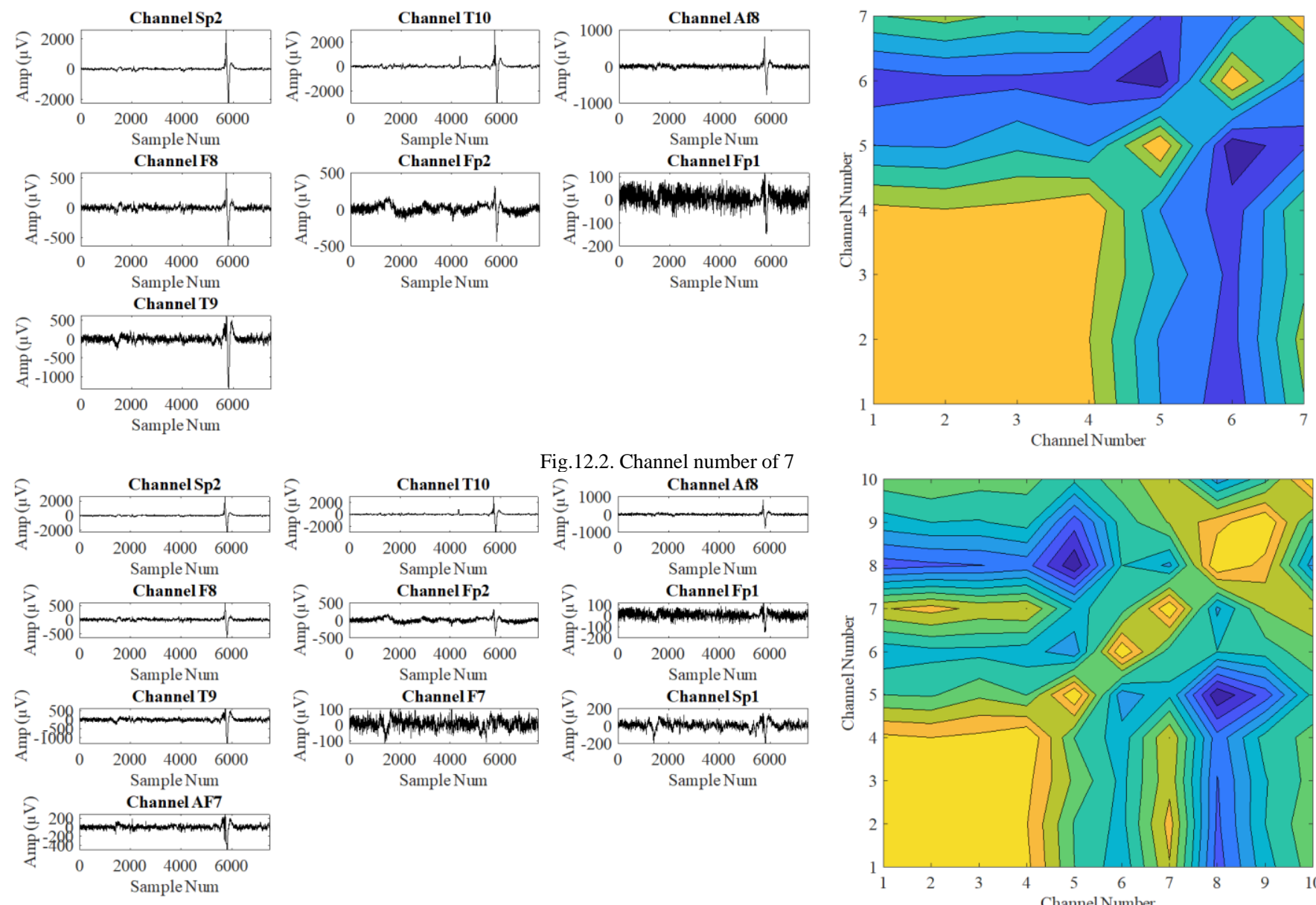

Fig.12.3. Channel number of 10

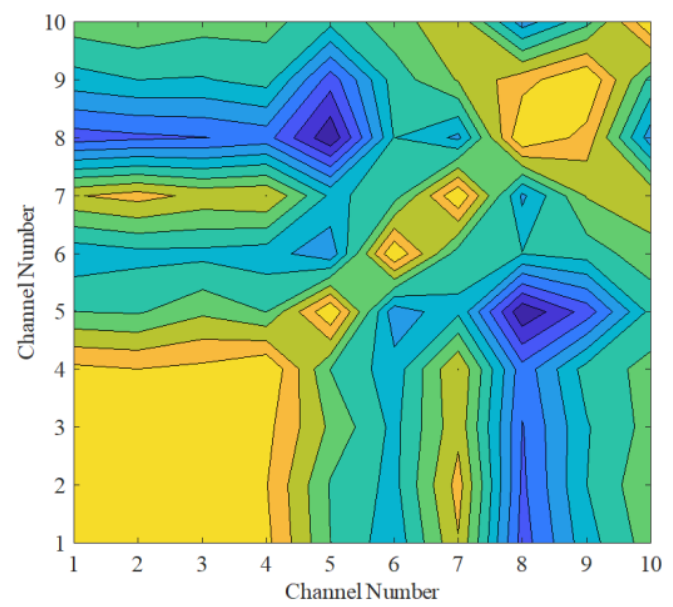

Fig.12. The effect of different channel numbers in patterns of correlation coefficient contour plot

\subsection{PERFORMANCE COMPARISON BETWEEN CORRELATION-BASED CNN AND SPECTOGRAM- BASED CNN}

The performance metrics comparison with hyperparameter details of CNN with two different inputs of correlation coefficient contour and spectogram are given in table 2 . These results include epoch number, iteration number, time elapsed, mini-batch accuracy, validation accuracy, and loss function value for the validation data. The number of epochs was chosen to be 5 over 500 iterations. The training data were shuffled before every epoch. Learning rate was reduced over epochs and its speed was updated the by decreasing the learning rate, multiplying it by a fractional learn-rate drop factor over a specific number of epochs. According to the table 2, the validation loss at 0.2813 for correlation-based $\mathrm{CNN}$ allows to conclude that this method has more generalization capability compared to the spectogram-based CNN [20, 21] with validation loss of 0.5143 .

TABLE II. DEEP LEARNING MODEL PERFORMANCE OVER OBSERVATIONS IN THE MINI-BATCH

\begin{tabular}{|c|c|c|c|c|c|c|c|c|c|c|c|c|}
\hline \multirow[b]{2}{*}{ 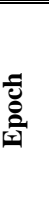 } & \multirow[b]{2}{*}{ } & \multicolumn{2}{|c|}{ Time Elapsed (hh:mm:ss) } & \multicolumn{2}{|c|}{ Mini-batch Accuracy } & \multicolumn{2}{|c|}{ Validation Accuracy } & \multicolumn{2}{|c|}{ Mini-batch Loss } & \multicolumn{2}{|c|}{ Validation Loss } & \multirow[b]{2}{*}{ تَّ } \\
\hline & & 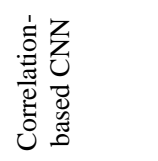 & 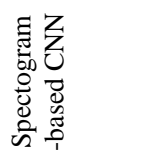 & 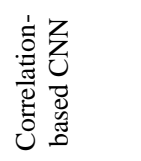 & 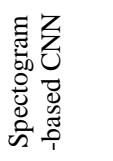 & 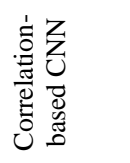 & 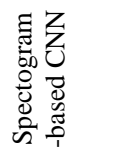 & 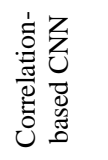 & 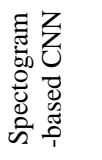 & 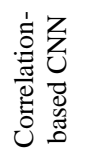 & 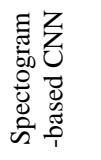 & \\
\hline 1 & 1 & 00:00:37 & $00: 00: 29$ & $65.62 \%$ & $62.50 \%$ & $49.71 \%$ & $49.19 \%$ & 0.7275 & 0.8091 & 8.0175 & 8.1011 & 0.0010 \\
\hline 1 & 50 & 00:03: & 00:03 & $93.75 \%$ & $75.00 \%$ & & & & 3.9856 & 3.2380 & 3.2711 & \\
\hline 1 & 100 & 00:06: & $00: 06$ & $93.75 \%$ & $87.50 \%$ & 88.46 & $81.93 \%$ & 0.6425 & 1.3046 & 0.9722 & 2.0516 & 010 \\
\hline 2 & 150 & 00:09 & & $87.50 \%$ & $78.12 \%$ & & $73.04 \%$ & 0.3596 & 0.3719 & 0.5752 & 1.6082 & 10 \\
\hline 2 & 200 & & & 93 & & & & & 0.2122 & & 0.78 & 10 \\
\hline 3 & 250 & & & 96.8 & & & 82 & 0.1191 & 0.2856 & 0.3504 & 0.6083 & 1.00 \\
\hline 3 & 300 & 0 & & 93.7 & & & & & 0.5239 & 0.3241 & 0.5469 & 1.000 \\
\hline 4 & 350 & 00:20: & $00: 21$ & $100.00 \%$ & $96.88 \%$ & 92.31 & $82.81 \%$ & 0.0228 & 0.0713 & 0.2988 & 0.5370 & $1.0000 \mathrm{e}-05$ \\
\hline 4 & 400 & $00: 23: 20$ & 00:24:18 & $93.75 \%$ & $87.50 \%$ & $92.09 \%$ & $82.52 \%$ & & 0.2218 & 0.2888 & 0.5294 & $1.0000 \mathrm{e}-05$ \\
\hline 5 & 450 & $00: 26: 10$ & 00:27:18 & $93.75 \%$ & $90.62 \%$ & $91.65 \%$ & $83.04 \%$ & 0.1153 & 0.2856 & 0.2814 & 0.5130 & $1.0000 \mathrm{e}-07$ \\
\hline 5 & 500 & $00: 28: 58$ & $00: 29: 47$ & $93.75 \%$ & $87.50 \%$ & $92.30 \%$ & $83.33 \%$ & 0.0227 & 0.3385 & 0.2813 & 0.5143 & $1.0000 \mathrm{e}-07$ \\
\hline \multicolumn{8}{|c|}{ Final Accuracy of CNN with input images of correlation coefficient contour } & \multicolumn{4}{|c|}{$92.30 \%$} & \\
\hline \multicolumn{8}{|c|}{ Final Accuracy of CNN with input images of spectogram } & \multicolumn{4}{|c|}{$82.81 \%$} & \\
\hline
\end{tabular}



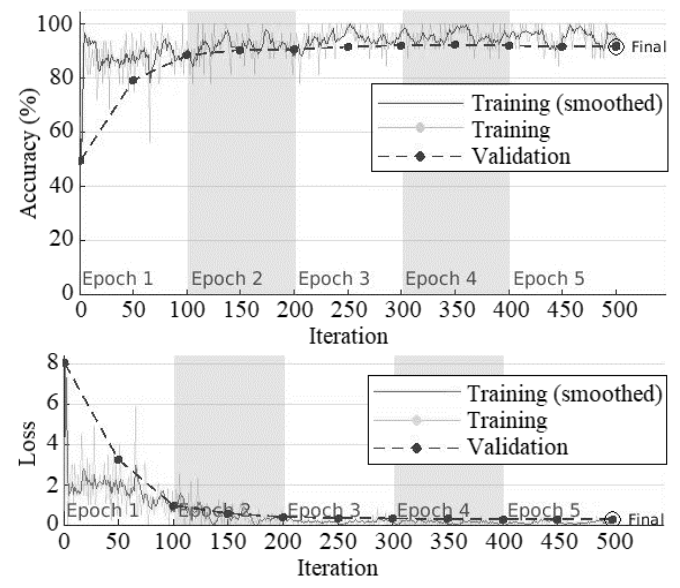

Fig.13. Accuracy and loss function variation in each epoch of correlationbased CNN (Validation accuarcy of $92.30 \%$, AUC of 0.96 )
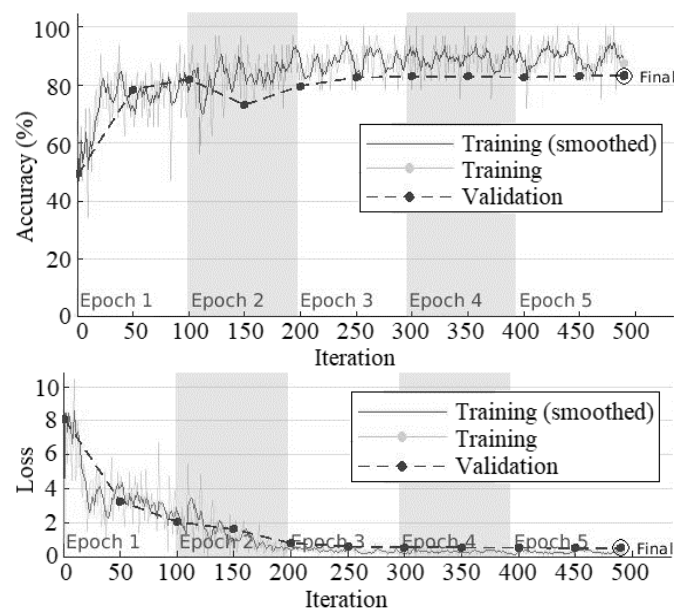

Fig.14. Accuracy and loss function variation in each epoch of spectogrambased CNN (Validation accuarcy of $82.81 \%$, AUC of 0.86 )

Convergence of average accuracy and loss function during training and validation for 5 epochs in both correlation-based and spectogram-based CNN were plotted in Figure 13 and 14. According to these results, the performance on the validation set for the case of $\mathrm{CNN}$ with input images of correlation coefficient contour is better than that of the validation set for the CNN with input images of spectogram. The result of the correlation-based model has rapidly converged to a stable value with no sign of overfitting.

\subsection{PERFORMANCE COMPARISON CORRELATION-BASED CNN AND EEGNet}

BETWEEN

For more evaluation, the performance of correlation-based and spectrogram-based $\mathrm{CNN}$ methods were compared against a compact convolutional neural network for EEG-based applications called EEGNet [22]. The EEGNet architecture given in figure 15 consists of a combination of temporal and special convolutions to learn special-frequency features and optimally mix them together. This EEGNet was fed by multichannel EEG data matrix of size $10 \times 7500$ and yielded to a validation accuracy of $81.25 \%$ with loss of 0.4400 after the elapsed time of $93 \mathrm{~min}$ and $48 \mathrm{~s}$ for the training process using single CPU (figure 16).

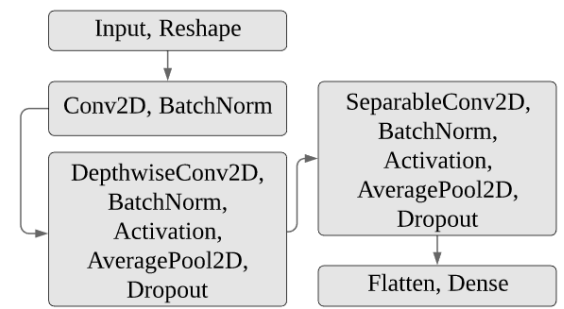

Fig. 15. EEGNet architecture
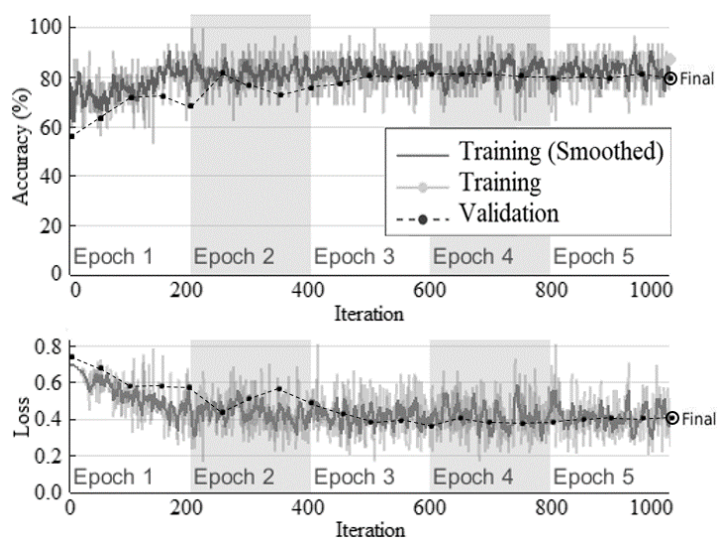

Fig. 16. EEGNet (Validation accuracy of $81.25 \%$, AUC of 0.90)

In order to analyze the validation phase of trained model, four metrics of accuracy, area under a ROC curve (AUC), sensitivity and specificity were compared with those achieved by two other control methods (Table 3).

TABLE III. EVALUATION METRICS OF TWO CONTROL METHODS

\begin{tabular}{cllll}
\multicolumn{5}{c}{ COMPARED WITH CORRELATION_BASED CNN } \\
\hline \hline Method & Accuracy & AUC & Sensitivity & Specificity \\
\hline \hline Correlation_based CNN & 92.30 & 0.95 & 100 & 91.7 \\
Spectogram-based CNN & 82.81 & 0.86 & 69.80 & 89.70 \\
EEGNet & 81.25 & 0.90 & 98.11 & 77.3 \\
\hline \hline
\end{tabular}

\subsection{THE EFFECT OF PARAMETER SELECTION ON THE} RESULTS OF CORRELATION-BASED CNN

Different scenarios were performed to evaluate the effect of parameters selection on the method performance. The results of some of these scenarios are summarized in following figures of 17 to 19 . The obtained results show that the validation accuracy slightly decreases when the number of isolines and number of channels are getting smaller.

\subsection{REAL-TIME APPLICATION}

In a different scenario, we aimed at investigating whether an accurate high-resolution image of correlation coefficient isoline map can be inferred from only correlation coefficient matrix at a far lower image resolution of $10 \times 10$ pixels (Figure $20)$. Isoline mapping is only an easy way of $2 \mathrm{D}$ upsampling, providing more resolution and thus more robust pattern to be learned. However, it doesn't provide more information. This can be explained by an increase in the complexity which results in higher computational burden, but at the same time greater accuracy as well. Therefore, it is only the matter of trade-off between size, computation and accuracy.

Figure 21 shows simplified CNN architecture which was trained to infer $10 \times 10$ images generated directly from correlation coefficient matrix. As illustrated in figure 22 and table 4, an acceptable accuracy was achieved for the low- 
resolution inputs, but with drastically reduced computation time.

In conclusion, although using 2D isoline map of correlation coefficient matrix yields lower validation loss, considering the proposed approach without extracting isoline map-based features reduces dramatically the complexity of the algorithm without losing almost relevant information and makes the approach feasible in real-time application by significantly getting faster.

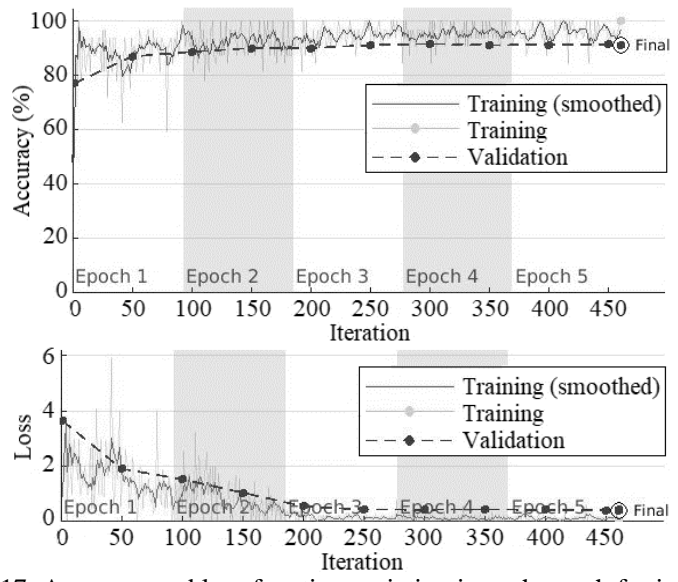

Fig.17. Accuracy and loss function variation in each epoch for isoline numbers of 5 (Validation accuracy of $91.36 \%$, AUC of 0.94 )
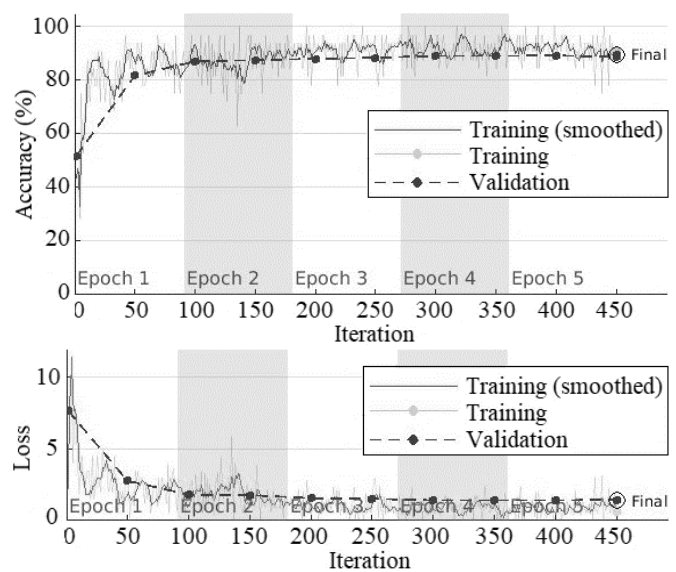

Fig.18. Accuracy and loss function variation in each epoch for isoline numbers of 3 (Validation accuracy of $89.57 \%$, AUC of 0.97 )
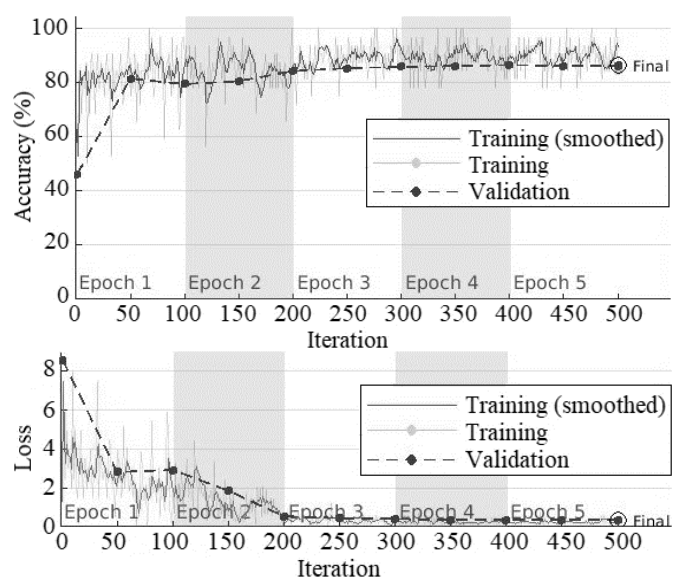

Fig.19. Loss function variation in each epoch for channel numbers of 73 (Validation accuracy of $86.31 \%$, AUC of 0.95 )
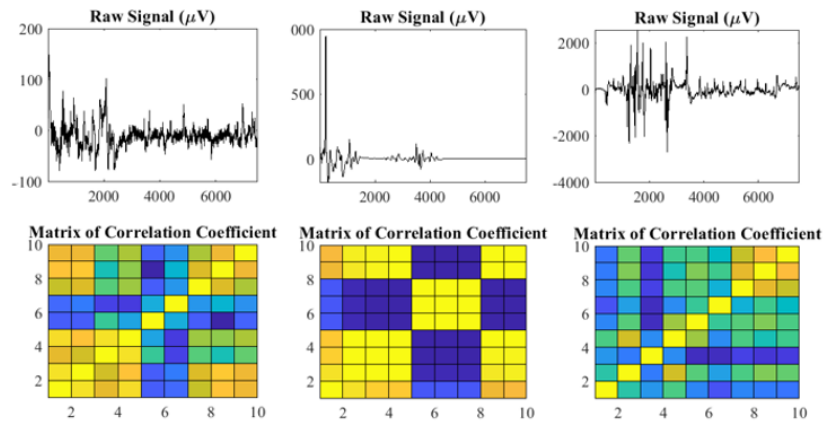

Fig. 20. 10×10 images of correlation coefficient

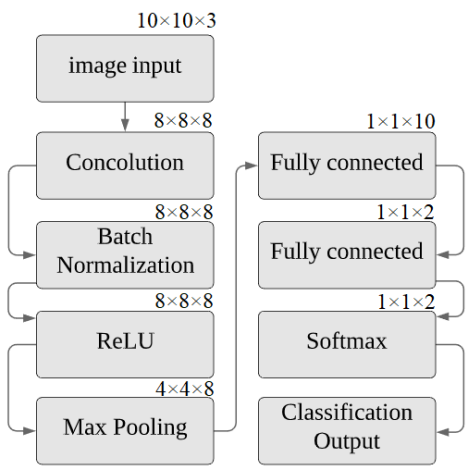

Fig. 21. Simplified CNN architecture for real-time application
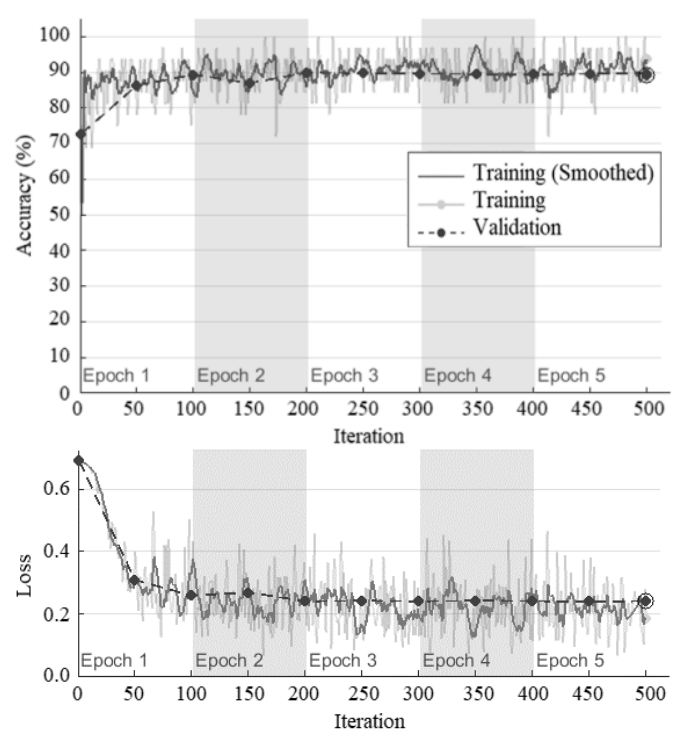

Fig. 22. Simplified CNN architecture performance in each epoch (Validation accuracy of $89.33 \%$ )

TABLE IV. COMPUTATION TIME WITH SIMPLIFIED MODEL

\begin{tabular}{ll}
\hline \hline Validation accuracy & $89.33 \%$ \\
Elapsed time for model training & $58 \mathrm{~s}$ \\
Hardware resource & Multiple CPUs \\
Iteration number & 500 \\
Latency for making real-time decision & $0.0181 \mathrm{~s}$ \\
\hline \hline
\end{tabular}

\section{CONCLUSION AND DISCUSSION}

We are facing upcoming transition from isolated environments and laboratories with minimum level of movement and noise to non-isolated environment with massive movements and external interferences likes ICU or even dailylife outdoor environment. This became possible with emergence of portable-wearable wireless EEG acquisition 
systems and headsets with few dry electrodes in recent years, replacing the large-sized heavy-weighted traditional acquisition systems with lots of electrodes attached to the whole scalp. In the ICU environment, there are a lot of external sources potentially causing artifacts to the EEG recordings. The ICU room is usually filled with electronic devices including motorized beds, blood pressure cuffs, pulse oximeters, catheters, pacemakers, extracorporeal membrane oxygenation devices, tissue oximetry monitors, intracranial pressure monitors, cooling/warming blankets, IV pumps and ventilators. Besides these electronic devices, the EEG in ICU environment is quite often contaminated by a wide range of physiological artifacts coming from voluntary body movement of facial expressions and eye blinks or involuntary movement like respiration, tremor as well as rigor and myoclonus. Most importantly, long duration of the EEG measurements in ICU makes the problem more challenging. Apart from continuous appearance of massive complex artifacts arising from nonisolated environments during recordings, dry electrodes may not provide the quality of recorded signals by traditional wet EEG electrodes because of electrode-skin impedance [24]. Considering above concerns, this paper presented an EEG artifact detection technique using the spatial correlation information in which the degree of spatial dependence among all EEG channels were simultaneously considered. The proposed method requires no auxiliary reference signal and no specific feature extraction. The method was shown to be suitable in detecting artifacts from recordings collected with an easy-to-use electrode and device suitable for demanding clinical environment such as ICU where electrode attachment with minimum preparation is highly appreciated [25].

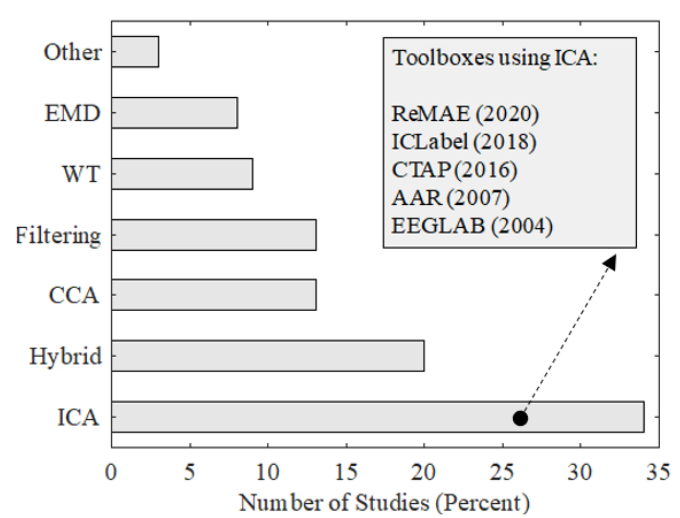

Fig. 23. Percentage comparison among commonly used artifact removal solutions

Figure 23 provides the bar chart comparing the percentage of works based on the artifact elimination techniques in the recent years. The figure is created using the results of [26, 27]. According to the figure, independent component analysis (ICA) is the most commonly used technique among all the approaches and also among most of EEG processing toolboxes which already been developed. One drawback of ICA-based artifact removal techniques is that they assume that sources should be statistically separated. Furthermore, extraction of some components based on the local optimization during the ICA procedure on EEG recordings may results in the artificial polarity indeterminacy on the projected component at some electrodes [28]. Adaptive filtering or regression methods also require the reference signals. Wavelet transform (WT) is not accurate in the case of overlapping with spectral properties. Mode-mixing may also cause empirical mode decomposition (EMD) to be not precise for artifact removal. Apart from these, none of these studies have offered a solution covering all types of artifacts. Therefore, there is a trend towards replacing traditional approaches with machine/deep learning algorithms for not only effectively removing the artifacts but also identifying numerous types of artifacts in the different scenarios.

Summary of some previous studies and comparison with the proposed method has been listed in Table 5. Even though the accuracies of these techniques are not comparable as they are not tested on the same dataset, these approaches are limited to the type of artifacts, and in this regards the proposed method could be said to be more effective compared to others. The obtained results also showed a classification accuracy of 92.30\% (AUC=0.96) in a leave-three-subjects-out crossvalidation procedure, representing high reliability of introduced method in detecting various types of artifacts, and being comparable to the approaches proposed earlier in the literature. The previous methods introduced in literature enable to detect only particular types of artifact while the proposed method can detect artifacts regardless of its type.

There are also some other differences between this study and previous ones. In [18], a deep learning-based artifact detection method has been introduced to automatically label 1-second segment of EEG signal which performs prediction faster and needs less computation time. However, the mentioned model was fit for more training epochs than proposed technique. In [29], 1-second sequence of EEG was used as well, which again made its method relatively fast. In [30] and [29], because of dividing a data set into shorter segments, more training samples were used compared to current study. In [31], noise with different powers was added to raw EEG signal to verify the effectiveness of artifact removal.

For more performance assessment, the findings were compared by applying EEGNet on the same dataset. The impact of input change to spectrogram on CNN performance was also explored. The results showed that the performance of CNN fed by correlation coefficient contour is better than that of CNN with spectogram as input as well as EEGNet fed by raw EEG sequence. Moreover, the result of CNN fed by spectogram get stagnant and even overfitted as the number of epochs increases.

In general, the advantages of current study are as follow:

o Visual representation of signal in temporal-spatial domain

o Visible difference in the correlation coefficient contour of two groups with and without artifact

o Simple network architecture

o Outperforming in accuracy in compare to EEGNet and spectrogram-based CNN

o No need for choosing spectral bands and channels

o No need for hand-picked features

o Automatically picking up the discriminative features 
without any need for tuning the network

o No need for using GPU

o Using the snapshot of EEG signals with different lengths

o Using only the forehead EEG signals

o No need for auxiliary reference signal

o Reducing dimension by fusing data in all channels

A major limitation of current work is the relatively small size of the dataset. Lack of interpretability of outcome obtained from deep learning network as a black box system is another limitation of this study.

Future research direction will includes classifying various types of artifacts based on their unique patterns in their correlation coefficient contour map, increasing the achieved accuracy by improving the architecture of deep network, using different regularization methods, using data augmentation techniques to increase dataset, normalizing input data and finally using larger dataset.

\begin{tabular}{|c|c|c|c|c|c|}
\hline Literatures & Artifact & Method & $\begin{array}{c}\text { Number of } \\
\text { channels }\end{array}$ & Accuracy & $\begin{array}{c}\text { Need for additional } \\
\text { recording }\end{array}$ \\
\hline$[18]$ & $\begin{array}{l}\text { Eye movements, Chewing, Shivering, } \\
\text { Electrode pop, Muscle artifacts }\end{array}$ & $\begin{array}{l}\text { Combination of CNN, RNN and } \\
\text { DCNN deep learning networks }\end{array}$ & 22 & $67.59 \%$ & - \\
\hline [30] & EOG & $\begin{array}{c}\text { Sparse autoencoder training } \\
\text { algorithm }\end{array}$ & 3 & $\simeq 90 \%$ & No \\
\hline [30] & EOG & $\begin{array}{l}\text { Sparse autoencoder training } \\
\text { algorithm }\end{array}$ & 1 & $\simeq 70 \%$ & No \\
\hline [31] & EOG & Convolution Neural Network & - & $80 \%$ & - \\
\hline [32] & Ocular artifact & $\begin{array}{c}\text { Multi-layer perceptrons (MLP) } \\
\text { neural networks }\end{array}$ & 2 & $72.92 \%$ & - \\
\hline [32] & Ocular artifact & $\begin{array}{c}\text { Stacked sparse autoencoder training } \\
\text { algorithm }\end{array}$ & 59 & $79.8 \%$ & No \\
\hline $\begin{array}{c}\text { Current } \\
\text { study }\end{array}$ & Different types of artifact & $\begin{array}{c}\text { Correlation-based Convolution } \\
\text { Neural Network }\end{array}$ & 10 & $92.24 \%$ & No \\
\hline
\end{tabular}

\section{REFERENCES}

[1] C. Chang, S. Hsu, L. Pion-Tonachini and T. Jung, "Evaluation of Artifact Subspace Reconstruction for Automatic Artifact Components Removal in Multi-Channel EEG Recordings," in IEEE Transactions on Biomedical Engineering, vol. 67, no. 4, pp. 1114-1121, April 2020.

[2] S. Lee, M. J. McKeown, Z. J. Wang and X. Chen, "Removal of HighVoltage Brain Stimulation Artifacts from Simultaneous EEG Recordings," in IEEE Transactions on Biomedical Engineering, vol. 66, no. 1, pp. 50-60, Jan. 2019.

[3] X. Li, C. Guan, H. Zhang and K. K. Ang, "Discriminative Ocular Artifact Correction for Feature Learning in EEG Analysis," in IEEE Transactions on Biomedical Engineering, vol. 64, no. 8, pp. 1906-1913, Aug. 2017.

[4] J. Metsomaa, J. Sarvas and R. J. Ilmoniemi, "Blind Source Separation of Event-Related EEG/MEG," in IEEE Transactions on Biomedical Engineering, vol. 64, no. 9, pp. 2054-2064, Sept. 2017.

[5] J. T. Valderrama, A. de la Torre, B. Van Dun, "An automatic algorithm for blink-artifact suppression based on iterative template matching: Application to single channel recording of cortical auditory evoked potentials," J. Neural Eng., vol. 15, no. 1, 016008, 2018.

[6] H. Gonzalo Rey, C. Pedreira, and R. Quian Quiroga, "Past, present and future of spike sorting techniques," In: Brain research bulletin 119, 106117, 2015.

[7] T. Brouns, "Automated signal processing for extracellular neural recordings," M.S. thesis, Radboud University, 2018.

[8] J. Jaeyoon Jun et al, "Real-time spike sorting platform for high-density extracellular probes with ground truth validation and drift correction," In: bioRxiv, 101030, 2017.

[9] E. Bak`stein et al., "Methods for automatic detection of artifacts in microelectrode recordings," Journal of neuroscience methods 290, 39-51, 2017.

[10] B. Singh and H. Wagatsuma, "A removal of eye movement and blink artifacts from EEG data using morphological component analysis," Comput. Math. Methods Med., vol. 2017, Jan. 2017, Art. no. 1861645, 2017.

[11] M. Chavez, F. Grosselin, A. Bussalb, F. De Vico Fallani, X. NavarroSune, "Surrogate-based artifact removal from single-channel EEG," IEEE Trans.Neural Syst.Rehab.Eng. 26, no.3, 540-550, 2018.

[12] J. Levitt, A. Nitenson, S. Koyama, L. Heijmans, J. Curry, JT. Ross, S. Kamerling, CY. Saab, "Automated Detection of Electroencephalography Artifacts in Human, Rodent and Canine Subjects using Machine Learning," Journal of Neuroscience Methods, 2018.
[13] P. Nejedly, J. Cimbalnik, P. Klimes et al., "Intracerebral EEG Artifact Identification Using Convolutional Neural Networks," Neuroinformatics. $1-10,2018$.

[14] S. ORegan, S. Faul, W. Marnane, "Automatic detection of EEG artefacts arising from head movements using EEG and gyroscope signals," Medical engineering physics, 35 (7): 867-874, 2013.

[15] M. Mohammadpour and V. Rahmani, "A Hidden Markov Model-based approach to removing EEG artifact," 2017 5th Iranian Joint Congress on Fuzzy and Intelligent Systems (CFIS), Qazvin, pp. 46-49, 2017.

[16] S.H ORegan, "Artifact detection and removal algorithms for EEG diagnostic systems," PhD Thesis, University College Cork, 2013.

[17] T. Radüntz, J. Scouten, O. Hochmuth, B. Meffert, "Automated EEG artifact elimination by applying machine learning algorithms to ICAbased features," J. Neural Eng. 14, 046004, 2017.

[18] D. Kim and S. Keene, "Fast Automatic Artifact Annotator for EEG Signals Using Deep Learning" IEEE SPMB 2019, Pennsylvania, USA, 2019.

[19] P. Nejedly, J. Cimbalnik, P. Klimes et al. "Intracerebral EEG Artifact Identification Using Convolutional Neural Networks," Neuroinform 17: $225,2019$.

[20]Q. Liu et al., "Spectrum Analysis of EEG Signals Using CNN to Model Patient's Consciousness Level Based on Anesthesiologists' Experience," in IEEE Access, vol. 7, pp. 53731-53742, 2019.

[21]D. Montilla-Trochez, R. Salas, A. Bertin, I. Griskova-Bulanova, P. Lisboa, C. Saavedra, "Convolutional neural network for cognitive task prediction from EEG's auditory steady state responses," CRoNe: 44-50, 2019.

[22] V. Lawhern, A.J. Solon, N.R. Waytowich, S.M. Gordon, C.P. Hung, B. Lance, "EEGNet: A Compact Convolutional Network for EEG-based Brain-Computer Interfaces," Journal of neural engineering, 15 5, 056013, 2018.

[23] S. Myllymaa and et. al., "Assessment of the suitability of using a forehead EEG electrode set and chin EMG electrodes for sleep staging in polysomnography.” J Sleep Res. 25(6): 636-45, 2016.

[24] J. Minguillon, M.A. Lopez-Gordo, F. Pelayo, "Trends in EEG-BCI for daily-life: Requirements for artifact removal," Biomed. Signal Process. Control. 2017.

[25] J. Kortelainen, E. Väyrynen, and et. al, "Forehead electrodes sufficiently detect propofol-induced slow waves for the assessment of brain function after cardiac arrest," J Clin Monit Comput., 2019.

[26] X. Jiang, G. B. Bian, Z. Tian, "Removal of Artifacts from EEG Signals: A Review," Sensors (Basel), vol. 19, no. 5, article 987, 18 p, 2019. 
[27] X. Chen et al., "ReMAE: User-Friendly Toolbox for Removing Muscle Artifacts From EEG," in IEEE Transactions on Instrumentation and Measurement, vol. 69, no. 5, pp. 2105-2119, 2020.

[28] F. Cong, I. Kalyakin, T. Ristaniemi, H. Lyytinen, "Drawback of ICA Procedure on EEG: Polarity Indeterminacy at Local Optimization," In: 14th Nordic-Baltic Conference on Biomedical Engineering and Medical Physics. IFMBE Proceedings, vol 20. Springer, Berlin, Heidelberg, 2008.

[29] B. Yang, K. Duan, C. Fan, C. Hu, J. Wang, "Automatic ocular artifacts removal in EEG using deep learning Biomed" Signal Process. Control 43, 148-58, 2018.

[30] B. Yang, K. Duan, T. Zhang, "Removal of EOG artifacts from EEG using a cascade of sparse autoencoder and recursive least squares adaptive filter" Neurocomputing 214, 1053-60, 2016.

[31] S. Wang, B. Guo, C. Zhang, X. Bai and Z. Wang, "EEG detection and denoising based on convolution neural network and Hilbert-Huang transform" Proc.-2017 10th Int. Congress on Image and Signal Processing, BioMedical Engineering and Informatics, pp 1-6, 2018.

[32] A. Drouin-Picaro, T.H. Falk, "Using deep neural networks for natural saccade classification from electroencephalograms" Proc. IEEE EMBS Int. Student Conf.: Expanding the Boundaries of Biomedical Engineering and Healthcare, pp 1-4, 2016. 